\title{
Milligram Morphine Equivalent
}

National Cancer Institute

\section{Source}

National Cancer Institute. Milligram Morphine Equivalent. NCI Thesaurus. Code C153260.

A value assigned to opioids to represent their relative potencies. It is determined by using an equivalency factor to calculate a dose of morphine that is equivalent to the ordered opioid. 